\title{
Observation on Clinical Therapeutic Effect, Toxic and Side Effects of Capecitabine plus Fuzheng Jiedu Decoction Supplemented and Reduced Prescription for Advanced Gastric Cancer
}

\author{
QUNYING ZOU AND WENTONG WU1*
}

Department of Medical Quality Management, 'Department of Oncology, Jinhua Hospital of Traditional Chinese Medicine, Jinhua, Zhejiang 321000, China

Zou et al.: Capecitabine plus Fuzheng Jiedu Decoction for Advanced Gastric Cancer

\begin{abstract}
This study deals with observation on clinical therapeutic effect and toxic and side effects of capecitabine plus Fuzheng Jiedu decoction supplemented and reduced prescription for advanced gastric cancer. From January 2011 to January 2014, 145 patients with gastric cancer diagnosed by surgery, gastroscopy and pathology in our hospital, with measurable tumor lesions, were enrolled in this study. They were aged 45-85 $\mathrm{y}$, with mean age (62.3 \pm 6.7$) \mathrm{y}, 75$ males and 70 females. In the study group, the disease progression time and survival time were 2-19.5 mo and 4.5-24 mo, respectively with 5 cases survived, while those of the control group were 1.5-20 months and 4.2-23 mo, respectively, with 3 cases survived. The t test showed no significant difference between the two groups $(\mathrm{p}>0.05)$. The survival rate of 60 cases within $1 \mathrm{y}$ in the study group $(87.0 \%)$ was significantly higher than that of the control group (59 cases, $77.9 \%)$, and the difference was significant $(\mathbf{p}<\mathbf{0 . 0 5})$. The incidence rates of hand-foot syndrome, nausea and vomiting, diarrhea, abdominal pain, liver injury and neurotoxicity in the study group were $21.7 \%, 2.9 \%, 11.6$ $\%, 7.2 \%, 1.4 \%$ and $17.4 \%$, which were significantly lower than that of the control group $(43.4 \%, 22.3$ $\%, 25 \%, 21.1 \%, 11.8 \%$ and $36.8 \%)$ and the chi-square test showed a significant difference $(\mathrm{p}<0.05)$. Capecitabine plus Fuzheng Jiedu decoction supplemented and reduced prescription for treating advanced gastric cancer has a good therapeutic effect, less toxic and side effects and the patient's quality of life is significantly improved. It is worthy of being popularized.
\end{abstract}

Key words: Capecitabine, Fuzheng Jiedu decoction, advanced gastric cancer

With the development of society and the change of human life style, digestive tract tumors, especially gastric cancer, become one of the major diseases that threaten human life and health ${ }^{[1]}$. As a fluorouracil-based oral cytotoxic agent, capecitabine is non-cytotoxic, easy to administer and has good efficacy against digestive tract tumors ${ }^{[2]}$.

However, in clinical application, oral capecitabine is easy to produce adverse reactions such as hand-foot syndrome, stomatitis and nausea and vomiting, which seriously affect patients quality of life ${ }^{[3]}$. Professor $\mathrm{Yu}$ Rencun $^{[4]}$ pointed out that the combination of chemotherapy and traditional Chinese medicine in the treatment of digestive tract tumors can reduce toxic and side effects and improve patients symptoms.

In this study, capecitabine plus Fuzheng Jiedu decoction supplemented and reduced prescription was used to treat patients with advanced gastric cancer with poor tolerance. The quality of life was improved after treatment with capecitabine plus Fuzheng Jiedu decoction supplemented and reduced prescription and the effect was good. It is reported below.

\section{MATERIALS AND METHODS}

General data:

From January 2011 to January 2014, 145 patients with 
gastric cancer diagnosed by surgery, gastroscopy and pathology in our hospital, with measurable tumor lesions, were enrolled in this study. They were aged $45-85 \mathrm{y}$, with mean age $(62.3 \pm 6.7) \mathrm{y}, 75$ males and 70 females. All patients were in stage VI according to international tumor $(\mathrm{T})$, nodes $(\mathrm{N})$ and metastases $(\mathrm{M})$ TNM classification of malignant tumors.

Performance status score (Karnofsky performance status scale (KPS) score)) was below 60 points in 9 cases, $61-70$ points in 78 cases, $71-80$ points in 47 cases and $81-90$ points in 11 cases and the predicted survival time was higher than 3 mo. All patients showed no contraindication to chemotherapy on blood routine examination, electrocardiography (ECG) and liver and kidney function examination and were given the same life care and health education during the treatment period.

According to the order of admission, the patients were divided into control group $(\mathrm{n}=76)$ and study group $(n=69)$. The former was treated with capecitabine alone and the latter with capecitabine and traditional Chinese medicine. There was no statistical difference in the basic data of gender, age and disease course between the two groups, which was comparable $(p>0.05)$. In addition, consent was obtained from the patient and his/ her family and informed consent was obtained.

\section{Methods:}

Study methods: All patients received oral capecitabine (trade name: Xeloda, purchased from Shanghai Roche Pharmaceutical Co., Ltd.) $1000 \mathrm{mg} / \mathrm{m}^{2}$, twice daily after $30 \mathrm{~min}$ meal for $14 \mathrm{~d}$, followed by $7 \mathrm{~d}$ off treatment, i.e. $21 \mathrm{~d}$ was considered as one treatment cycle. Each patient was orally administered for at least 2 cycles. The blood routine and liver and kidney function were examined before and after the completion of chemotherapy and the therapeutic effect and toxic and side effects were observed and evaluated.

Treatment plan of traditional Chinese medicine: Chinese medicine Fuzheng Jiedu decoction was used in this group. Its basic formula was Codonopsis pilosula 15-20 g, Astragalus membranaceus 15-20 g, Rehmannia glutinosa 10-15 g, Radix paeoniae Alba 10-15 g, Curcuma zedoary 15 g, Rhizoma polygonati 12-15 g, edible tulip $15 \mathrm{~g}$, Salvia chinensis $15 \mathrm{~g}$, raw oyster $30 \mathrm{~g}$, Scutellaria barbata $30 \mathrm{~g}$ and endothelium corneum gigeriae galli $10 \mathrm{~g}$. Since oral administration of capecitabine, Fuzheng Jiedu decoction was orally administered, twice/d, until the end of chemotherapy course.
Combine the side effects of capecitabine and the patient's constitution, add and subtract the components as appropriate: add $10 \mathrm{~g}$ of XuanHuSuo, $10 \mathrm{~g}$ of Curcuma sichuanensis and $10 \mathrm{~g}$ of Aspongopus for abdominal pain patients; subtract Rehmannia glutinosa and add $10 \mathrm{~g}$ of stir-baked Dioscoreae rhizoma, $10 \mathrm{~g}$ of Aspongopus and $10 \mathrm{~g}$ of Poria cocos for diarrhea patients; add $10 \mathrm{~g}$ of ginger processed Pinellia, $10 \mathrm{~g}$ of Pericarpium citri reticulatae, $6 \mathrm{~g}$ of Alpinia katsumadai Hayata, $6 \mathrm{~g}$ of Amomi Fructus for nausea patients; add $15 \mathrm{~g}$ of Alismatis rhizoma, $6 \mathrm{~g}$ of Cassia twig, $15 \mathrm{~g}$ of pericarpium Arecae for abdominal effusion patients; add $15 \mathrm{~g}$ of Radix Ophiopogonis, $15 \mathrm{~g}$ of Scrophulariae radix, $15 \mathrm{~g}$ of glossy privet fruit for patients with yin deficiency, epigastric noise and red tongue with little moss; add $12 \mathrm{~g}$ of Fructus Psoraleae, $12 \mathrm{~g}$ of Herba Epimedii and $12 \mathrm{~g}$ of Fructus Evodiae for patients with yang deficiency and fear of cold, vomit and deep pulse; add $20 \mathrm{~g}$ of cortex dictamni, $20 \mathrm{~g}$ of radix Sophora flavescens, $20 \mathrm{~g}$ of Zaocys dhumnades and $20 \mathrm{~g}$ of Caulis spatholobi for patients with severe hand-foot syndrome.

Investigation indicators: The short-term efficacy (change of tumor body), patient's quality of life, longterm efficacy and toxic and side effects were used as indicators to evaluate the therapeutic efficacy. The short-term efficacy was evaluated by the change of tumor body. After the completion of chemotherapy, reexamination of magnetic resonance imaging (MRI) or computed tomography (CT) was performed to observe the size of solid tumor according to response evaluation criteria in solid tumors (RECIST) ${ }^{[5]}$. It can be divided into progression (PD), stability (SD), partial response (PR) and complete response (CR). The effective rate of $\mathrm{PR}+\mathrm{CR}$ and disease control rate of $\mathrm{PR}+\mathrm{CR}+\mathrm{SD}$ were calculated.

According to Karnofsky (KPS) scoring system ${ }^{[6]}$, the quality of life score can be divided into improvement (difference higher than 10 points), stability (difference between 10 and -10 points) and reduction (difference lower than -10 points) according to the difference of scores before and after treatment. Long-term efficacy; the time of disease progression and survival time of all patients were calculated from the start of chemotherapy to the end of death. The survival rates within $1 \mathrm{y}$ were observed and compared between the two groups. Toxic and side effects: With reference to the acute and subacute toxic reaction grading standard established by World Health Organization (WHO) $)^{[7]}$, they were classified as grades 0-IV. 


\section{Statistical processing:}

Statistical package for the social sciences (SPSS) 17.0 was used for data analysis, $\chi^{2}$ test was used for comparison of counting data and test was used for comparison of measuring data. $\mathrm{p}<0.05$ was used for statistically significant results.

\section{RESULTS AND DISCUSSION}

Comparison of recent efficacy was done below. The effective rate and disease control rate were $30.4 \%$ and $68.1 \%$ in study group and $25 \%$ and $51.3 \%$ in control group, respectively. Chi-square test showed no significant difference between the two groups $(\mathrm{p}>0.05)$ (Table 1).

Quality of life comparison was done. The quality of life was improved in $59.5 \%$ (41 cases) of the study group. Chi-square test showed that the KPS score of the study group after treatment was significantly higher than that before treatment and in the control group and the difference was significant $(\mathrm{p}<0.05)$ (Table 2).
Comparison of long-term efficacy is carried out (fig. 1). The disease progression time and survival time of the study group were 2-19.5 mo and 4.5-24 mo, respectively, with 5 cases survived, while those of the control group were 1.5-20 mo and 4.2-23 mo, respectively, with 3 cases survived. The t test showed that there was no significant difference between the two groups ( $>00.05$ ). The survival rate of 60 cases in the study group $(87.0 \%)$ was significantly higher than that of the control group (59 cases, $77.9 \%$ ) within $1 \mathrm{y}$ and the difference was significant $(\mathrm{p}<0.05)$.

Comparison of adverse reactions was done. The incidence of hand-foot syndrome, nausea and vomiting, diarrhea, abdominal pain, liver injury and neurotoxicity in the study group was $21.7 \%, 2.9 \%, 11.6 \%, 7.2 \%$, $1.4 \%$ and $17.4 \%$, which was significantly lower than that in the control group $(43.4 \%, 22.3 \%, 25 \%, 21.1 \%$, $11.8 \%$ and $36.8 \%$ ). Chi-square test showed significant difference $(\mathrm{p}<0.05)$ (Table 3).

TABLE 1: COMPARISON OF RECENT EFFICACY

\begin{tabular}{lccccccc}
\hline Group & $\mathrm{n}$ & $\mathrm{CR}$ & PR & SD & PD & Effective rate (\%) & Disease control rate (\%) \\
\hline Study group & 69 & 1 & 20 & 26 & 22 & (21) 30.4 & $(47) 68.1$ \\
Control group & 76 & 2 & 17 & 20 & 37 & $(19) 25$ & $(39) 51.3$ \\
$\chi^{2}$ & & & & & 2.13 & 2.01 & 0.057 \\
$\mathrm{p}$ & & & & & & 0.051 & \\
\hline
\end{tabular}

TABLE 2: COMPARISON OF KPS SCORES OF QUALITY OF LIFE BETWEEN THE TWO GROUPS

\begin{tabular}{lccc}
\hline Group & $\mathrm{n}$ & Before treatment & After treatment \\
\hline Study group & 69 & $62.1 \pm 5.1$ & $83.5 \pm 5.9^{* \#}$ \\
Control group & 76 & $61.2 \pm 5.4$ & $58.9 \pm 4.8$ \\
\hline
\end{tabular}

Note: Intra-group comparison, ${ }^{*} \mathrm{p}<0.05$; inter-group comparison, ${ }^{*} \mathrm{p}<0.05$

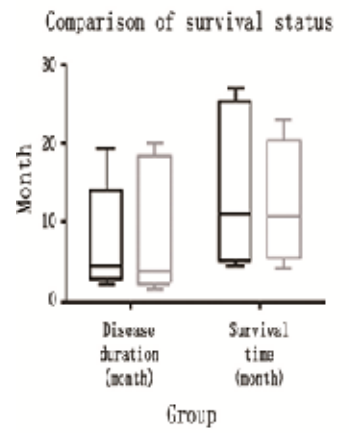

$\wedge$

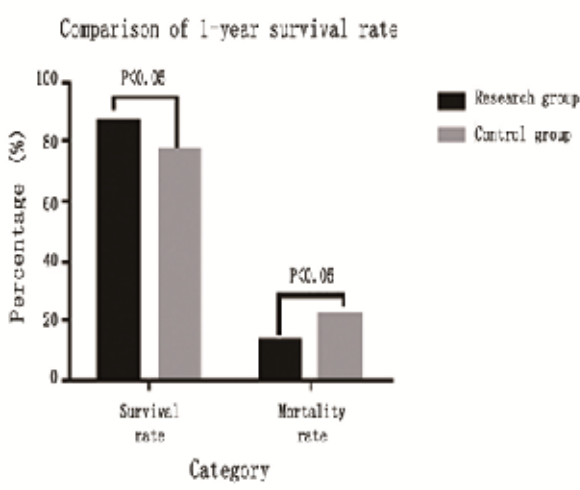

$\mathbf{B}$

Fig. 1: Comparison of long-term efficacy. (A) Comparison of survival time of patients and (B) Comparison of survival rate within $1 \mathrm{y}$. (ㅁ) Research group, ( $\mid \square$ ) Control group, ( $\square$ ) Research group, ( $\mid=$ ) Control group 
www.ijpsonline.com

TABLE 3: COMPARISON OF INCIDENCE OF TOXIC AND SIDE EFFECTS

\begin{tabular}{|c|c|c|c|c|c|c|c|c|c|c|c|c|}
\hline \multirow[b]{2}{*}{ Toxic and side effect } & \multicolumn{6}{|c|}{ Control group (76 cases) } & \multicolumn{6}{|c|}{ Study group (69 cases) } \\
\hline & $\begin{array}{c}\text { Grade } \\
0\end{array}$ & $\begin{array}{c}\text { Grade } \\
\text { I }\end{array}$ & $\begin{array}{c}\text { Class } \\
\text { II }\end{array}$ & $\begin{array}{c}\text { Class } \\
\text { III }\end{array}$ & $\begin{array}{c}\text { Class } \\
\text { IV }\end{array}$ & $\begin{array}{c}\text { Incidence } \\
\text { (\%) }\end{array}$ & $\begin{array}{c}\text { Grade } \\
0\end{array}$ & $\begin{array}{c}\text { Grade } \\
\text { I }\end{array}$ & $\begin{array}{c}\text { Class } \\
\text { II }\end{array}$ & $\begin{array}{c}\text { Class } \\
\text { III }\end{array}$ & $\begin{array}{l}\text { Class } \\
\text { IV }\end{array}$ & $\begin{array}{c}\text { Incidence } \\
\text { (\%) }\end{array}$ \\
\hline Hand-foot syndrome & 43 & 26 & 7 & 0 & 0 & 43.4 & 54 & 12 & 3 & 0 & 0 & $21.7^{*}$ \\
\hline Nausea and vomiting & 59 & 13 & 4 & 0 & 0 & 22.3 & 67 & 2 & 0 & 0 & 0 & $2.9^{*}$ \\
\hline Diarrhea & 57 & 11 & 8 & 0 & 0 & 25 & 61 & 8 & 0 & 0 & 0 & $11.6^{*}$ \\
\hline Abdominal pain & 60 & 12 & 4 & 0 & 0 & 21.1 & 64 & 5 & 0 & 0 & 0 & $7.2^{*}$ \\
\hline Liver and kidney injury & 67 & 5 & 4 & 0 & 0 & 11.8 & 68 & 1 & 0 & 0 & 0 & $1.4^{*}$ \\
\hline Electrocardiogram & 66 & 6 & 4 & 0 & 0 & 13.1 & 61 & 6 & 2 & 0 & 0 & 11.6 \\
\hline Neurotoxicity & 48 & 22 & 6 & 4 & 0 & 36.8 & 57 & 8 & 4 & 0 & 0 & $17.4^{*}$ \\
\hline
\end{tabular}

Note: Compared with control group, ${ }^{*} \mathrm{p}<0.05$

Gastric cancer is the most common type of malignant tumor, with the fourth highest incidence and the second highest mortality rate. In China, 360000 people die of gastric cancer every year ${ }^{[8]}$. Therefore, exploring an effective treatment for advanced gastric cancer is of great importance in improving quality of life and quality of survival ${ }^{[9]}$. Capecitabine is a fluoropyrimidine anti-cancer drug.

The oral therapeutic effect of capecitabine alone is significantly higher than that of 5-fluorouracil intravenous injection. The concentration of capecitabine in tumor tissue is significantly higher than that in normal tissue and capecitabine has good anti-tumor effect on drug-resistant cell lines.

Okines et al. reported that the efficacy rate of oral capecitabine in the treatment of advanced gastric cancer was up to $24 \%$. Therefore, oral capecitabine is an option when patients are unwilling to receive combination chemotherapy or are poorly tolerated ${ }^{[10]}$. In clinical studies, the most significant adverse reaction of oral capecitabine was hand-foot syndrome ${ }^{[11]}$. Tang et al. noted that the incidence rate of capecitabine handfoot syndrome was $44.4 \%$; Mainour et al. ${ }^{[12]}$ found that the adverse reactions of capecitabine were significantly related to the oral dose: the incidence rate of handfoot syndrome was $100 \%$ at the dose of $2500 \mathrm{mg}$ and reduced to $77.08 \%$ at the dose of $2000 \mathrm{mg}$. Therefore, to avoid significant adverse reactions, the selection of reasonable dose of capecitabine was particularly critical.

In addition to selecting appropriate doses, traditional Chinese medicine is also a comprehensive treatment for tumors, which has significant advantages in overcoming the adverse effects of chemotherapeutic drugs ${ }^{[13]}$.
Traditional Chinese medicine's understanding of the pathogenesis of cancer is divided into two aspects: vital qi weakness and domination of pathogen ${ }^{[14]}$.

Most of the patients in this group had advanced gastric cancer and stomach as the basis of after birth. Therefore, weakness of spleen and stomach, stagnation of toxic stasis and deficiency of qi and blood were considered as the pathogenesis. Therefore, Astragalus membranaceus, Atractylodis macrocephalae rhizoma and Codonopsis pilosula were chosen to supplement $\mathrm{qi}^{\left[{ }^{[15]}\right.}$, Radix paeoniae Alba, Rhizoma polygonati and Rehmannia glutinosa to nourish blood ${ }^{[16]}$, Salvia chinensis, Curcuma zedoary and raw oyster to invigorate the circulation of blood, Scutellaria barbata and edible tulip to disinfect ${ }^{[17]}$. The addition and decrease of the components with the disease are beneficial to relieving qi and stopping vomiting, with the effect of nourishing Yin and reinforcing Yang, so it can achieve the effect of strengthening the body and detoxifying and reduce the toxic and side effects.

In this study, the efficacy of capecitabine combined with traditional Chinese medicine in the treatment of patients with gastric cancer was comparable to that of capecitabine alone. There was no significant difference in the patient's survival time. However, the patient's quality of life was significantly improved and the incidence rate of toxic and side effects was lower than that of capecitabine combined with traditional Chinese medicine Fuzheng Jiedu decoction alone. It indicated that the combination of capecitabine and traditional Chinese medicine Fuzheng Jiedu decoction in the treatment of patients with advanced gastric cancer who were difficult to tolerate and had poor physical condition could indeed reduce the adverse drug reactions and high quality of life improvement and disease control 
rates were achieved.

In this study, according to the patient's toxic and adverse symptoms supplemented and reduced prescription, for syndrome differentiation combined with syndrome differentiation and differentiation, it is an effective exploration for the target treatment of tumor by the combination of traditional Chinese and western medicine. This is consistent with the principle and effect of Cunren et al. combined with Yiqi Huoxue traditional Chinese medicine and chemotherapy preparation in treatment of advanced digestive tract tumors ${ }^{[18]}$.

In this study, by observing the changes of various indicators, Fuzheng Jiedu decoction supplemented and reduced prescription could effectively improve the clinical yield of patients and significantly improve the toxic and side effects of capecitabine, including handfoot syndrome, vomiting and other adverse reactions, indicating that the combined treatment with traditional Chinese medicine has definite anti-toxic and synergistic effects. Therefore, capecitabine plus Fuzheng Jiedu decoction supplemented and reduced prescription for treatment of advanced digestive tract tumors has good therapeutic effect, less toxic and side effects and the patient's quality of life has been significantly improved, thus deserving to be widely used. However, it is necessary to expand the sample to further observe and confirm the advantages of integrated traditional Chinese and western medicine in the treatment of gastric cancer.

\section{Conflict of interests:}

The authors declared no conflict of interest.

\section{REFERENCES}

1. Fan CZ, Chu YP, Dai H. Comparative study on capecitabine combined with oxaliplatin and tegafur combined with oxaliplatin in treatment of advanced gastric cancer. J Clin Oncol 2011;16(8):725-7.

2. Liu H, Li Y, Ning ZQ. Efficacy and related factors of docetaxel combined with capecitabine in the treatment of recurrent metastatic breast cancer. J Clin Oncol 2011;16(10):905-8.

3. Liu X, Zhou ZF, Chen L. Clinical observation of paclitaxel combined with oxaliplatin and capecitabine in treatment of advanced gastric cancer. J Clin Oncol 2010;15(11):1023-5.

4. Tang L, Shen J, Wu M. Clinical observation of capecitabine combined with vinorelbine in the treatment of recurrent metastatic breast cancer. J Clin Oncol 2010;15(1):67-9.

5. Li H, Qin S, Hua H. Clinical observation of gemcitabine combined with fluorouracil in treatment of drug-resistant advanced colorectal cancer. J Clin Oncol 2010;15(9):811-4.

6. Cao W, Guo ZG. Clinical observation on prevention and treatment of capecitabine-associated hand-foot syndrome by traditional Chinese medicine. Oncol Res Clin 2012;24(9):6368.

7. Yin Q, Huang YC. Clinical observation of three-dimensional conformal radiotherapy combined with capecitabine chemotherapy for postoperative recurrence of rectal cancer. Oncol Res Clin 2010;22(5):335-7.

8. Li Lanman. Docetaxel combined with capecitabine in the treatment of 30 patients with metastatic breast cancer. Oncol Res Clin 2011;23(9):628-9.

9. $\mathrm{Lu} \mathrm{H}, \mathrm{Zhou} \mathrm{L,} \mathrm{Ju} \mathrm{J.} \mathrm{Comparison} \mathrm{of} \mathrm{short-term} \mathrm{efficacy} \mathrm{of}$ tegafur and capecitabine combined with oxaliplatin in the firstline treatment of advanced colorectal cancer. Cancer Res Clin Oncol 2012;24(9):613-5.

10. Qin D, Bing H, Keping S. Study on the combination of anticancer traditional Chinese medicine with five drugs. Chin J Exp Formul 2010;16(13):232-5.

11. Yaodong Z, Yanqing L. Study on suppression of tumor epithelial interstitial transformation by traditional Chinese medicine. Chin J Exp Formul 2014;20(6):228-32.

12. Yafei X, Shu Y. Research progress on anti-tumor mechanism of Paris polyphylla. Chin J Exp Formul 2012;18(9):304-7.

13. Passoni P, Reni M, Cattaneo GM, Slim N, Cereda S, Balzano $\mathrm{G}$, et al. Hypofractionated image-guided IMRT in advanced pancreatic cancer with simultaneous integrated boost to infiltrated vessels concomitant with capecitabine: a phase I study. Int J Radiat Oncol Biol Phys 2013;87(5):1000-6.

14. Perri F, Muto P, Argenone A, Ionna F, Longo F, Fulciniti $\mathrm{F}$, et al. Induction chemotherapy with docetaxel, cisplatin and capecitabine, followed by combined cetuximab and radiotherapy in patients with locally advanced inoperable squamous cell carcinoma of the head and neck: a phase I-II study. Oncology 2013;84(4):251-4.

15. Yan S, Jiang X, Yang J, Yan D, Wang YX. Radiotherapy for nasopharyngeal carcinoma and combined capecitabine and nimotuzumab treatment for lung metastases in a liver transplantation recipient: a case experience of sustained complete response. Cancer Biother Radiopharm 2012;27(8):519-23.

16. Wheeler HE, Gonzalez-Neira A, Pita G, de la Torre-Montero JC, Alonso R, Lopez-Fernandez LA, et al. Identification of genetic variants associated with capecitabine-induced handfoot syndrome through integration of patient and cell-line genomic analyses. Pharmacogenet Genomics 2014;24(5):2317.

17. Pierga JY, Bidard FC, Cropet C, Tresca P, Dalenc F, Romieu G, et al. Circulating tumor cells and brain metastasis outcome in patients with HER2-positive breast cancer: the LANDSCAPE trial. Ann Oncol 2013;24(12):2999-3004.

18. Jin K, Lan H, Xie B, He K, Xu Z, Li G, et al. Antitumor effects of FP3 in combination with capecitabine on PDTT xenograft models of primary colon carcinoma and related lymphatic and hepatic metastases. Cancer Biol Ther 2012;13(9):737-44.

This is an open access article distributed under the terms of the Creative Commons Attribution-NonCommercial-ShareAlike 3.0 License, which allows others to remix, tweak, and build upon the work non-commercially, as long as the author is credited and the new creations are licensed under the identical terms

This article was originally published in a special issue,
"Diagnostic and Therapeutic Advances in Biomedical Research
and Pharmaceutical Sciences"
Indian J Pharm Sci 2021:83(5) spl issue "11-15"

